\title{
Enhancement of T cell-independent immune responses in vivo by $\mathrm{CD} 40$ antibodies
}

\author{
Per Dullforce, Debbie C. Sutton \& Andrew W. Heath \\ Division of Molecular and Genetic Medicine and Sheffield Institute for Vaccine Studies, F Floor, \\ University of Sheffield Medical School, Beech Hill Road, Sheffield, S1O 2RX, UK \\ Correspondence should be addressed to P.D. or A.H.
}

In this report we describe a potentially powerful method for vaccinating infants against encapsulated bacterial pathogens such as Haemophilus influenzae, Streptococcus pneumoniae and Neisseria meningitidis. High levels of antibody directed against the polysaccharides of the bacterial capsule are normally protective $^{1-3}$. Unfortunately, the capsular polysaccharides are $\mathbf{T}$ cellindependent antigens ( $\mathrm{TI}$ ); lacking T-cell help, they induce only weak, predominantly IgM antibody responses, with infants responding especially poorly ${ }^{4}$. T-cell help, given to B cells during responses to protein antigens, causes stronger antibody responses and isotype switching to the IgG isotypes. T-cell help is mainly mediated through ligation of the B-cell surface antigen, CD40, by its cognate T-cell ligand, CD154 (ref. 5-9). Here we show that administering anti-CD40 monoclonal antibody to mice, along with pneumococcal polysaccharide, provides a substitute for T-cell help and results in the generation of strong, isotype-switched antibody responses, which are protective. The work points the way toward a possible effective and inexpensive means of protecting susceptible groups against important bacterial pathogens.

Immune responses to capsular polysaccharide from $S$. pneumoniae were studied using a murine model. Intraperitoneal immunization of BALB/c mice with type 3 pneumococcal capsular polysaccharide (PS3) alone induced weak IgM and IgG3 responses against the antigen (Fig. 1a). This is typical of the response to TI type II antigens in mice (humans produce IgM and IgG2). Administration of the anti-CD40 antibodies 1 C10 or 4F11 with PS3 induced small but significant rises in specific IgM and IgG3, whereas, remarkably, 1C10 induced significant polysaccharidespecific IgG1, IgG2a and IgG2b responses. These isotypes are not normally seen in response to TI type II antigens. The $1 \mathrm{C} 10$ antibody would appear to have successfully mimicked T-cell help by inducing high antibody titers and isotype switching in vivo. The anti-polysaccharide response was extremely persistent, with antibody being detected at high titers 14 weeks after the single immunization (Fig. 1a). No memory response against the polysaccharide was induced, as a second injection of polysaccharide alone failed to boost antibody responses (data not shown).

Streptococcus pneumoniae has more than 80 different capsular polysaccharide types, and any vaccination would be expected to induce protective immunity against a number of the more common serotypes ${ }^{3}$. A current pneumococcal vaccine, Pneumovax II (Merck, Sharp and Dohme), consists of 23 different polysaccharides. Mice were immunized with this 23 -valent vaccine and $1 \mathrm{C} 10$. Inclusion of the CD40 antibody successfully generated strong IgG responses against randomly chosen polysaccharide types 4, 8, 12 and 19 (Fig. 2). Such isotype-switched responses were also generated against the two other antigens we examined, types 3 and 14, and the pattern of IgG isotype distribution against the polysaccharide was similar to that shown in Fig. $1 a$ (data not shown). Therefore, 1C10 enhances responses to TI type II antigens other than PS3.

Given that administration of CD40 antibody mixed with polysaccharide would not restrict or even target CD40 ligation to antigen-specific $\mathrm{B}$ cells, we anticipated polyclonal activation of $\mathrm{B}$ cells with a resultant rise in total serum immunoglobulin levels. Indeed, 1C10 and PS3 induced some splenomegaly and 2- to 4fold rises in total serum immunoglobulin levels (Fig. 1b). This, however, should be contrasted with up to 50-fold rises in specific antibody levels, indicating that polysaccharide-specific antibody production was preferentially enhanced. This skewing toward specific antibody is also not unexpected as it reflects in vitro findings. In vitro, although $1 \mathrm{C} 10$ could induce B-cell proliferation in the absence of stimulation through the antigen receptor, proliferation was synergistically enhanced by such costimulation ${ }^{10}$. The 4F11 antibody, which largely lacks agonist activity in vitro, did not enhance responses as efficiently as 1C10, demonstrating an association between adjuvant activity in vivo and B-cell activation in vitro.

Although induction of polyclonal antibody responses is not in itself a major problem, these responses may increase the risk of autoantibody production. Serum samples taken from mice treated with $500 \mu \mathrm{g}$ of $1 \mathrm{C} 1014$ days or 14 weeks earlier were screened against Hep- 2 cells for autoantibodies. No autoantibodies were detected, except in the positive control serum from MRL mice (data not shown). However, we cannot rule out the risk of autoantibody production. Any potential risk should be greatly reduced by lowering the dose of CD40 stimulator, and we have achieved some enhancement of specific immune responses against PS3 with doses as low as $50 \mu \mathrm{g} 1 \mathrm{C} 10$. We would envisage that the dose could be reduced much further by efficient colocalization of antigen and stimulator, perhaps through coemulsification or coentrapment in microparticles or liposomes. Indeed, in separate work, administering a $\mathrm{T}$ cell-dependent antigen attached to $1 \mathrm{C} 10$ has allowed the stimulatory dose of antibody to be cut from $500 \mu \mathrm{g}$ to below $1 \mu \mathrm{g}$, while maintaining marked enhancement of specific immune responses without polyclonal activation (manuscript in preparation).

During a T cell-dependent response, CD40 ligation is necessary for switching to IgG isotypes, but various cytokines also play important roles ${ }^{8,9,11-13}$. It was, therefore, intriguing that such isotype-switched responses were obtained without the addition of exogenous cytokines. This suggests either that CD40 and antigen receptor ligation may be sufficient to induce isotype switching or that bystander cells may provide sufficient cytokines to switch 
Fig. 1 CD40 antibodies induce $\mathbf{a}$, enhanced class-switched antibody responses to PS3 and $\boldsymbol{b}$, increased total serum immunoglobulin. Mean logarithmic titers are displayed for serum samples taken, days 7 and 14 and week 14 after $\mathrm{BALB} / \mathrm{c}$ mice were injected with PS3 and 1C10, $4 \mathrm{~F} 11$ or isotype control antibody GL117. The IgM and IgC isotype mean logarithmic titers are shown when they were maximal, respectively, days 7 and 14 after injection and also at 14 weeks. All negative results were given a logarithmic titer of 20 , the lowest dilution used. Statistical significance compared with the relevant $\mathrm{GL} 117$ control by Student's $t$-test, ${ }^{*} P<0.05$.

the activated B cells in vivo ${ }^{12,14,15}$ We considered that the CD40 antibodies might be stimulating T-cell cytokine production, either directly through ligation of CD40 on $\mathrm{T}$ cells $\mathrm{s}^{16,19}$ or indirectly through induction of costimulatory molecules on B cells or other antigen-presenting cells ${ }^{17-19}$. The $1 \mathrm{C} 10$ antibody does induce high levels of costimulatory molecules, whereas $4 \mathrm{~F} 11$ has no effect (ref. 10 and unpublished data). The action of $4 \mathrm{~F} 11$ showed T-cell dependency, as it failed to augment polysaccharide-specific responses in CD4-depleted mice (Fig. 3). However, $1 \mathrm{C} 10$ and PS3 administration induced a pronounced, isotypeswitched response in CD4-depleted mice (Fig. 3), with IgG responses to polysaccharide being better than those induced in normal mice, demonstrating a CD4-independent action. Similar results were obtained when athymic nude mice were used instead of CD4-depleted mice (data not shown).

Most vaccines under development for use against encapsulated bacteria are protein-polysaccharide conjugates, which aim to provide $T$-cell help for the anti-polysaccharide response through $\mathrm{T}$-cell recognition of epitopes on the protein. By their nature, such conjugates are not as effective in CD4-deficient patients such as those with AIDS (ref. 20,21). In contrast, the use of a CD40 stimulator would not only avoid the high cost of conjugate production, but as we have shown, would generate responses unaffected by a CD 4 deficiency.

The major fault with capsular polysaccharide only vaccines is that infants and young children, while reacting normally to TD antigens, respond poorly to TI type II antigens. Indeed, children under 2 years old fail to respond at all to many TI type II antigens ${ }^{22-25}$ The inability of their immune systems to act against bacterial capsules correlates with increased susceptibility to infection. They are the group most in need of effective vaccines. $\mathrm{CBA} / \mathrm{N}$ (xid) mice have an X-linked immunodeficiency rendering them, like infants, unable to respond to TI type II antigens ${ }^{26}$. Although one report has stated otherwise ${ }^{27}$, in our hands, $B$ cells

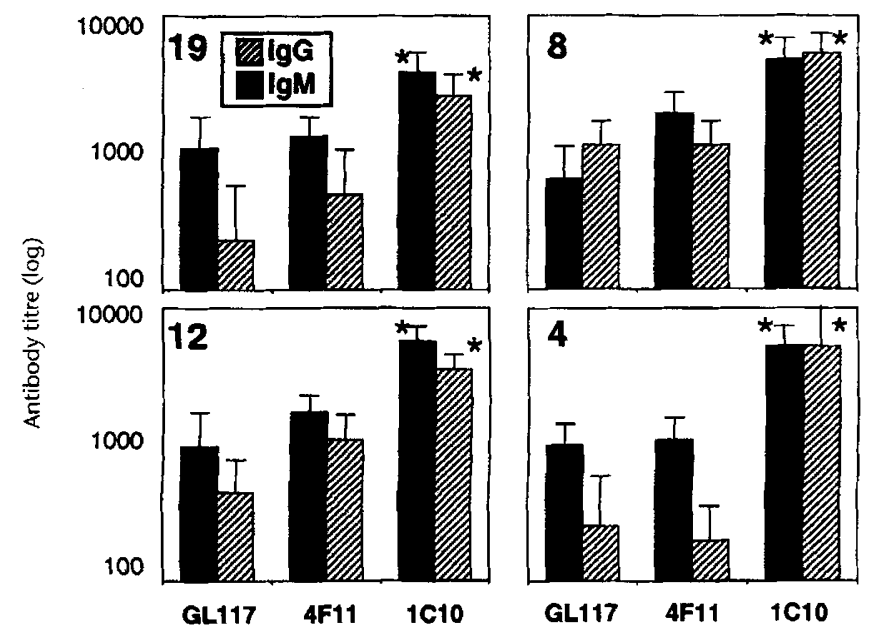

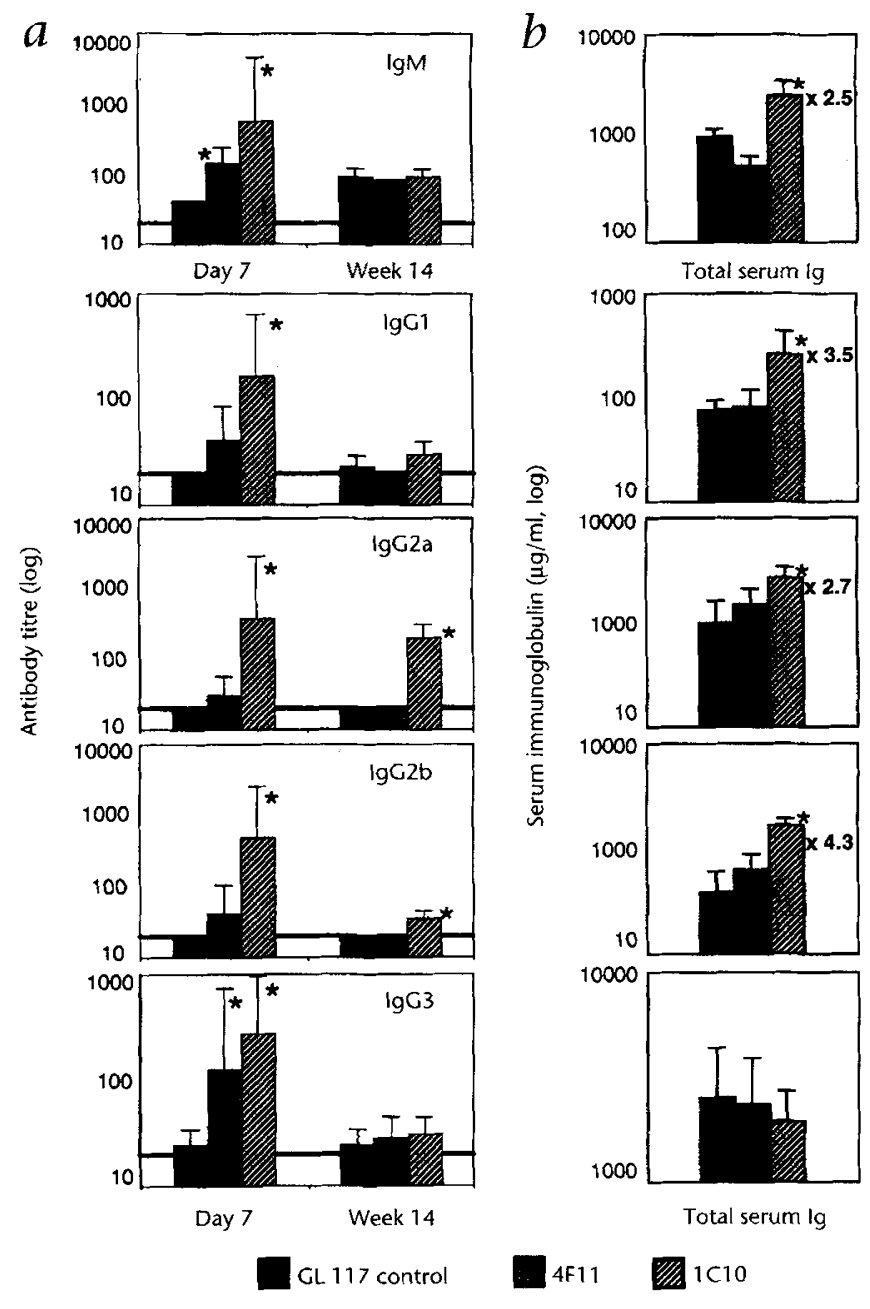

from $\mathrm{CBA} / \mathrm{N}$ mice react normally to $\mathrm{CD} 40$ ligation in vitro (ref. 28 and unpublished data A.H.). We immunized groups of xid mice with $1 \mathrm{C} 10$ plus PS3 and successfully generated IgG2a and IgG2b responses against PS3 (Fig. $4 a$ ). Thus, the B-cell defect in these mice was successfully by-passed by administering the CD 40 antibody as an adjuvant along with antigen.

Using the mouse model system, we have shown that CD40 stimulators can enhance the antibody response to pneumococcal polysaccharides, producing greater antibody levels and the production of IgG isotypes. As do protein-polysaccharide conjugates, $1 \mathrm{C} 10$ can induce polysaccharide-specific responses in xid mice, which like infants are unable to respond to vaccines based on polysaccharide only. Unlike protein-polysaccharide conjugates, the adjuvant action of $1 \mathrm{C} 10$ is $\mathrm{CD} 4^{+}$cell-independent, which is a definite advantage for the vaccination of patients with CD4 deficiencies, for example, AIDS sufferers ${ }^{20,21}$. One potential problem with the use of CD40 stimulators as adjuvants may be autoantibody production. We believe the risk should be minimized by improved delivery of antigen and CD40 stimulator. Finally, while 1C10

Fig. 2 Antibody responses to other pneumococcal polysaccharides are also enhanced by CD40 antibody. IgM and IgG responses to types 19, 8, 12 and 4. 5. pneumoniae capsular polysaccharides in mice 10 days after immunization with the 23 capsular polysaccharides in Pneumovax 11 and either the CD40 antibody 1 C10 or control antibody GL117. All the 1 C10 responses were significantly different from the GL117 responses by Student's $t$-test, ${ }^{\star} P<0.05$. 
Fig. 3 The mechanism of $1 \mathrm{C10}$ action is $\mathrm{CD}^{+} \mathrm{T}$ cell-independent. PS3specific antibody logarithmic titers (day 14) were induced in CD4-depleted $\mathrm{BALB} / \mathrm{c}$ mice treated i.p. with $\mathrm{PS} 3$ and $1 \mathrm{C} 10,4 \mathrm{~F} / 1$ or control antibody GL117. All 1 C10 responses were significantly different from the relevant GL117 control by Student's t-test, $* p<0.05$.

administered with PS3 clearly enhances specific antibody responses, the measure of a vaccine is whether it provides long-term protection against disease. We challenged mice, immunized 9 months previously, with $10^{5}$ colony-forming units (CFU) of $S$. pneumoniae type 3 (Fig. $4 b$ ), Of the BALB/c mice administered with PS3 and 1C10,5 of 8 survived challenge, whereas only 1 of 6 and none of 11 mice survived in the groups receiving, respectively, PS 3 with GL117 and PS3 alone (chi-square test, $P<0.05$ ).

The CD40 stimulators, such as antibodies, recombinant soluble CD154, or molecular mimics of CD154, have considerable potential as immunological adjuvants for $\mathbf{T}$ cell-independent antigens.

\section{Methods}

Mice and materials. The mice used were $\mathrm{BALB} / \mathrm{c}$ mice (in house), $\mathrm{CBA} / \mathrm{ca}$ and CBA/N (xid) mice (Harlan-Olac UK, Bicester). They were 6-12 weeks old at the start of the experiments. The pneumococcal capsular polysaccharides type 1, 3, 4, 8, 12, 13, 19 and 23 were obtained from American Type Culture Collection (ATCC, Rockville, MD), preumococcal cell wall polysacçaride from Statens Serum Institut (Copenhagen, Denmark) and Pneumovax II vaccine from Merck, Sharp and Dohme (Hoddesden, UK). The anti-CD40 antibodies, $1 \mathrm{C} 10$ and 4F11 (ref. 10), with their isotypematched rat lgG2a control antibody GL117, were purified by Sheffield Hybridomas (Sheffield, UK).
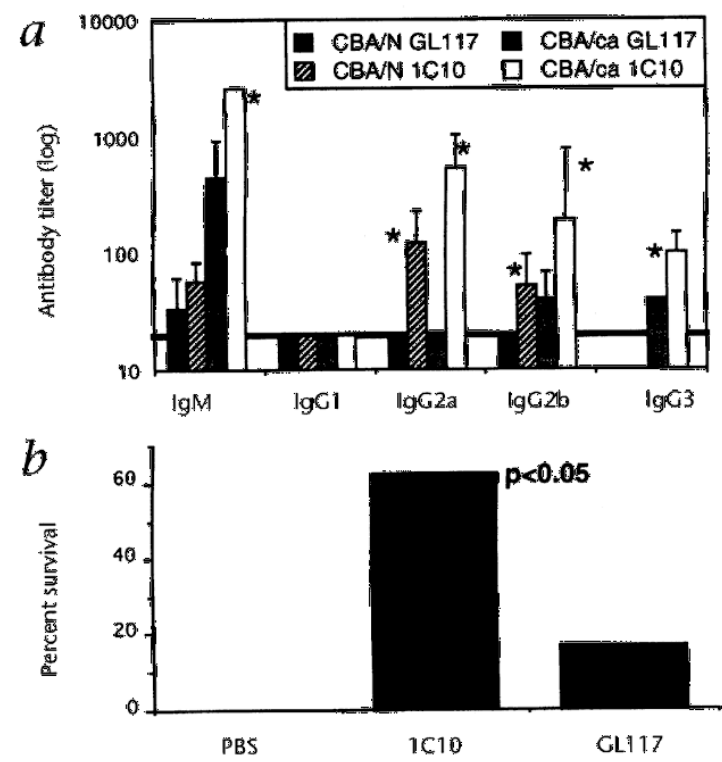

Fig. 4 CD 40 antibodies induce responses to PS3 in normally unresponsive xid mice $(a)$. Enhanced responses in BALB/C mice provide protection against $S$. pneumonioe challenge 9 months after treatment (b). a, PS3-specific antibody responses in CBA/N (xid) mice and control CBA/cä mice injected with PS3 and $1 C 10$ or GL117. The $\lg M$ and $\lg C$ isotype logarithmic titers shown are when they were maximal, respectively, day 7 and day 14 after injection. Statistical significance compared with the relevant GL117 control (Student's t-test, ${ }^{*} P<0,05$ ). b. Percentage survival in $B A L B / c$ mice challenged with $S$. pheumonice type 3 , but 9 months previously administered PS3 and $1 C 10$, GL117 or PBS. There were 8,6 and 11 mice in the $1 \mathrm{C} 10, \mathrm{GL} 117$ and PBS treated groups, respectively. Survivall in the $1 \mathrm{C} 10$ group was significantly enhanced compared with the control groups (chi= square test, $P<0.05$ ).

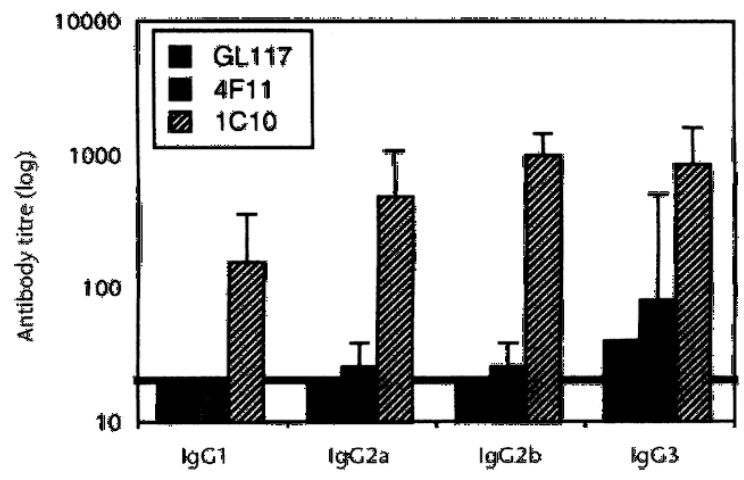

Immunization protocols. Mice were treated with $500 \mu \mathrm{g}$ of $1 \mathrm{C} 10,4 \mathrm{~F} 11$ or GL117 and $20 \mathrm{ng}$ PS3 i.p., except those receiving Pneumovax II. BALB/C mice receiving Pneumovax II were injected i.p. with $500 \mu \mathrm{g}$ of either $1 \mathrm{C} 10$ or GL117 and $1 / 25$ th of the recommended human dose of Preumovax 11. This equates to $1 \mu \mathrm{g}$ of each of the 23 polysaccharides present in the vaccine. At least five mice were used for each experimental group.

Experiment in CD4-depleted mice. BALB/C mice, 6-10 weeks old, were depleted of CD4 cells 5 days before the start of the experiment. Depleting anti-CD4 antibody VT\$ $191.1(500 \mu \mathrm{g})$ was injected intravenously and again the next day intraperitoneally. The percentage of $\mathrm{CD} 4{ }^{+}$splenocytes in the depleted mice as detected by flow cytometry had dropped to undetectable levels when the antibody and PS3 were injected. There was no IgG antibody response of these mice to $50 \mu \mathrm{g}$ of keyhole limpet hemocyanin, a T cell-dependent antigen (data not shown).

Measurement of polysaccharide antibodies and total serum immunoglobulin by ELISA. First, 96-well ELISA plates (Costar, High Wycombe, UK) were coated overnight with $10 \mu \mathrm{g} / \mathrm{ml}$ polysaccharide or with a $1 / 200$ dilution of anti-mouse immunoglobulin serum (Sigma, Poole, UK). Individual serum samples were titrated on the plates, and the various isotypes were detected by horseradish peroxidase (HRP)-conjugated mouse isotype-specific serum (Southern Biotechnology Associates, Birmingham, AL). Serum obtained from mice injected with Pneumovax II was absorbed against 5 . pneumoniae cell wall polysaccharide as described previously ${ }^{29}$. Antibodies to cell wall polysaccharide, a contaminant of all capsular polysaccharide preparations, might have created false-positive results. Total serum immunoglobulin concentrations were calculated with reference to calibrated mouse serum (Sigma). With the polysaccharide results end-point titers for each mouse were assessed against normal mouse serum, and then logarithmic mean titers and standard deviation were calculated. All negative results were given a logarithmic titer of 20 , the lowest dilution used.

Challenge with $S$. pneumonice. BALB/c mice were immunized 9 months before challenge with $20 \mu \mathrm{g}$ PS3 and $500 \mu \mathrm{g} 1 \mathrm{C10}$ i.p. Challenge was $10^{5}$ CFU of encapsulated S. pneumonicae type 3 (ATCC) given i.p. Numbers of surviving animals were ascertained 2 weeks after challenge.

\section{Acknowledgments}

We would like to thank Nicole Parish and Anne Cooke (Cambridge, UK) for generous donation of YTS 191.1.1, DNAX Research Institute for providing $1 C 10,4511$ and GL117 hybridomas, Anne Cooke, Andrew Mckenzie and Sarah Bell for reading the manuscript; and the Wellcome Trust for funding this work (grant number 042251).

\section{RECEIVED $12 \mathrm{MARCH}$; ACCEPTED 21 OCTOBER 1997}

1. Robbins, J.B.; Schneerson, R. \& Szu, S.C. Hypothesis: Serum IgCr antibody is sufficient to confer protection against infectious diseases by inactivating the inoculum. 1. Infect. Dis. 171, 1387-1398 (1995).

2. Landesiman, S.H. \& Schiffman, G. Assessment of the antibody response to pneumococcal vaccine in high risk populations. Rey. Intect. Dis. 3, \$1184-\$197 (1981).

3. Alonso De Velasco, E, Verheul, A.F.M., Verhoef, I. \& Snippe, H. Streptococcus prect- 
moniae: Virulence factors, pathogenesis and vaccines. Microbiol. Rev. 59, 591-603 (1995).

4. Mond, J.J., Lees, A. \& Snapper, C.M. T-cell-independent antigens type-2. Annu. Rev. Immunol. 13, 655-692 (1995).

5. Armitage, R.J. et al. Molecular and biological characterization of a murine ligand for CD40. Nature 357, 80-82 (1992).

6. Renshaw, B.R. et al. Humoral immune responses in CD40 ligand-deficient mice. I Exp. Med. 180, 1889-1900 (1994).

7. Castigli, E. et al. CD40-deficient mice generated by recombination-activating gene2-deficient blastocyst complementation. Proc. Natl. Acad. Sci. USA 91, 12135-12139 (1994)

8. Kawabe, $\mathrm{T}$. et al. The immune-response In CD40-deficient mice-impaired im munoglobulin class switching and germinal center formation. immunity 1 , 167-178 (1994).

9. Xu, J. et al. Mice deficient for the CD40 ligand. Immunity 1, 423-431 (1994).

10. Heath, A.W., Wu, W.W. \& Howard, M.C. Monoclonal antibodies to murine CD40 define two distinct functional epitopes. Eur. /. Immunol. 24, 1828-1834 (1994).

11. Coffman, R.L., Lebman, D.A. \& Rothman, P. Mechanism and regulation of immunoglobulin isotype switching. Adv. Immunol. 54, 229-270 (1993).

12. Warren, W.D. \& Berton, M.T. Induction of germ-line gamma-1 and epsilon ig gene expression in murine $B$ cells: IL-4 and the CD 40 ligand-CD40 interaction provide distinct but synergistic signals. I. Immunol. 155, 5637-5646 (1995).

13. Kuhn, R., Rajewsky, K. \& Muller, W. Generation and analysis of interleukin-4 deficient mice. Science 254, 707-710 (1991).

14. Schultz, C.L. et al. T helper cell membranes promote IL-4-independent expression of germ-line C-gamma-1 transcripts in B cells. J. Immunol. 149, 60-64 (1992)

15. Ferlin, W.G. et al. CD 40 signaling induces interleukin-4-independent lgE switching in vivo. Eur. f. Immunol. 26, 2911-2915 (1996).

16. Armitage, R.J. et al. CD40 ligand is a T cell growth factor. Eur. J. Immunol. 23, 2326-2331 (1993).

17. Kennedy, M.K. et al. Induction of B cell costimulatory function by recombinant murine CD40 ligand. Eur. I. Immunol. 24, 116-123 (1994)

18. Yang, Y.P. \& Wilson, J.M. CD40 ligand-dependent T cell activation: Requirement of
B7-CD28 signaling through CD40. Science 273, 1862-1864 (1996).

19. Fanslow, W.C. et al. Recombinant CD40 ligand exerts potent biologic effects on $T$ cells. J. Immunol. 152, 4262-4269 (1994).

20. Ahmed, F. et al. Effect of human immunodeficiency virus type 1 infection on the antibody response to a glycoprotein conjugate pneumococcal vaccine: Results from a randomised trial. J. infect. Dis. 173, 83-90 (1996).

21. Carson, P.I., Schut, R.L., Simpson, M.L., O'Brien, I. \& Janoff, E.N. Antibody and subclass responses to pneumococcal polysaccharides following immunization of human immunodeficiency virus-infected patients. 1. Infect. Dis. 172, 340-345 (1995).

22. Rijkers, G.T., Sanders, E.A.M., Breukels, M.A. \& Zegers, B.J.M. Responsiveness of infants to capsular polysaccharides: Implications for vaccine development. Rev. Med. Microbiol, 7, 3-12 (1996).

23. Lee, H.-1. et al. Immunogenicity and safety of a 23 -valent pneumococcal polysaccharide vaccine in healthy children and in children at increased risk of pneumococcal infection. Vaccine 13, 1533-1538 (1995).

24. Käyhty, H., Karanko, V., Peltola, H. \& Mäkelä, P.H. Serum antibodies after vaccination with Haemophilus influenzae type b capsular polysaccharide and responses to immunization: No evidence of immunologic tolerance or memory. Pediatrics 74 , 857-865 (1984).

25. Douglas, R.M., Paton, J.C., Duncan, S.J. \& Hansman, D.f. Antibody response to pneumococcal vaccination in children younger than five years of age. $)$. Infect. Dis. 148, 131-137 (1983).

26. Scher, I. CBA/N immune defective mice: Evidence for the failure of a $B$ cell subpopulation to be expressed. Immunol. Rev, 64, 117-136 (1982).

27. Hasbold, I. \& Klaus, G.G.B. B Cells From CBA/N mice do not proliferate following ligation of $C D 40$. Eur. I. Immunol. 24, 152-157 (1994).

28. Santos-Argumedo, L. et al. CD38 unresponsiveness of xid B cells implicates Bruton's tyrosine kinase (btk) as a regulator of $C D 38$ induced signal transduction. Int. Immunol. 7, 163-170 (1995).

29. Konradsen, H.B., Sorensen, U.B.S. \& Henrichsen, J. A modified enzyme-linked immunosorbent assay for measuring type-specific anti-pneumococcal capsular polysaccharide antibodies. 1. Immunol. Methods 164, 13-20 (1993). 